\title{
Can lymphadenectomy be omitted in advanced ovarian cancer? -a brief review
}

\author{
Rosa A. Salcedo-Hernández \\ Surgical Oncology, Gynecologic Tumors Division, Instituto Nacional de Cancerología, Mexico City, Mexico \\ Correspondence to: Rosa A. Salcedo-Hernández, MSc, MBHA. Surgical Oncology, Gynecological Tumors Division, Instituto Nacional de Cancerología \\ de México (Mexico's National Cancer Institute)., Av. San Fernando \# 22, Sección XVI, Tlalpan, Mexico City, Mexico. Email: rosasalher@gmail.com.
}

\begin{abstract}
The indication of systematic lymphadenectomy in advanced ovarian cancer without apparent macroscopic lymph node involvement has been controversial over the past three decades, and the recommendation to perform it or not has been based on multiple retrospective studies, small cohort studies, and few randomized studies with several biases; however, it seems that this controversy has come to an end after the recent publication of a randomized clinical trial. The study of lymph node disease in ovarian cancer has intensified in the last two decades, so far that it was part of the changes of the last update of the International Federation of Gynecology and Obstetrics (FIGO) staging; In this review, a search was made of the available literature to understand the evolution of knowledge about the implications of the realization or not of lymphadenectomy in two scenarios of advanced ovarian cancer (namely, the presence or not of lymph node disease macroscopic), without losing the landscape of the importance of peritoneal disease in these stages, which, as we will see throughout the review, the complete cytoreduction of the tumor remains an integral part of the treatment, since residual disease is one of the most relevant prognostic factors. Nowadays, we can confidently state that systematic lymphadenectomy in patients with advanced ovarian cancer without clinically apparent nodal disease is not necessary, and the presence of macroscopic retroperitoneal lymph node disease should be resected as part of cytoreductive surgery since it will be this and the residual disease that determine the prognosis of the patients.
\end{abstract}

Keywords: Ovarian cancer; lymph node dissection; surgery

Submitted Jan 21, 2020. Accepted for publication May 08, 2020.

doi: $10.21037 / \mathrm{cco}-20-35$

View this article at: http://dx.doi.org/10.21037/cco-20-35

\section{Introduction}

Ovarian cancer occurs in advanced stages in $75 \%$ to $80 \%$ of cases. The main route of dissemination and presentation of the disease is peritoneal, however, the lymphatic disease is common. The study of lymph node disease in ovarian cancer has intensified in the last two decades, so far that it was part of the changes of the last update of the International Federation of Gynecology and Obstetrics (FIGO) staging (2014), where stage III with exclusive lymph node involvement $(<10 \%)$ was substaged (IIIA) because it was observed that it has a better prognosis with respect to those who also have peritoneal disease (1). Also, in patients with advanced disease until recently there was controversy about the value of systematic lymphadenectomy in patients without apparent lymph node disease, changing this from the results of the LION study, leaving in another scenario patients with macroscopic nodal disease, where lymph node resection is part of the cytoreduction. The complete cytoreduction of the tumor remains an integral part of the treatment, since residual disease is one of the most relevant prognostic factors.

Since there are differences in lymph node disease in the advanced stages and because of this can be microscopic or macroscopic, we will develop the different considerations for these scenarios, without losing sight of the fact that in these stages the intraperitoneal disease will determine the extent of the surgery and the prognosis. In addition, 
another point under consideration is that ovarian cancer is a group of histological subtypes, each with different clinical behavior, which also means that lymph node involvement and indication of lymphadenectomy is influenced by the histological subtype.

\section{Microscopic lymph node disease}

In advanced ovarian cancer, a lymph node metastasis has been reported between $43 \%$ and $55.7 \%$ during systematic lymphadenectomy performed in these patients (2-4). Due to this high percentage of lymph node involvement, for a long time it was considered that performing a systemic lymphadenectomy allowed the complete resection of the disease allowing adequate staging and avoiding leaving residual disease, so it was prognostic and thought also therapeutic (5). In addition, as a result of studies of lymph node recurrence patterns and findings of second-look surgeries in patients with advanced ovarian cancer where residual lymph node disease was evidenced, it was theorized that the lymph node was a pharmacological sanctuary in which chemotherapy does not have adequate effectiveness as in the rest of the organism, allowing the disease to persist (6).

In the $90 \mathrm{~s}$, several retrospective studies reported improvement in survival in patients who underwent lymphadenectomy accompanied or not of adequate cytoreduction, however, these studies had different biases, including patient selection (presence or absence of macroscopic lymph node disease, better functional status, etc.), limited number thereof, the extent of surgery and lymphadenectomy, residual disease, chemotherapy scheme, which makes it unlikely to have reliable conclusions (7-9).

The first randomized study was reported in the 2000s; this study reported improvement in progressionfree survival, without improvement in overall survival in patients with advanced ovarian cancer with cytoreduction, randomized to lymphadenectomy vs non-lymphadenectomy; however, in the control arm, resection of the suspicious nodal disease was allowed, resection completeness was with residual disease larger than $1 \mathrm{~cm}$ and only one-third of the patients managed to leave without a visible intraperitoneal disease, limiting the impact of this result (10). Another study based on data from the SEER (Surveillance, Epidemiology and End Results) of a total of 13,918 patients, reported an improvement in survival by increasing the number of resected nodes: however, despite the number of patients, the lack of data as an extension of the disease and of the lymphadenectomy, as well as residual disease only allowed hypothesis generation (11).

The study by Du Bois et al. showed an improvement in survival in patients who underwent retroperitoneal lymphadenectomy, but this benefit was not maintained if there was macroscopic residual disease, which confirmed the greater importance of peritoneal disease. In addition to the fact that data used for this analysis were obtained from three different studies, whose objective was not to evaluate lymphadenectomy, different chemotherapy schemes were used, the patient group was heterogeneous and the study was retrospective in nature (12).

With these studies and subsequent meta-analyses $(13,14)$, a conclusive recommendation could not be given due to the heterogeneity of the information, which did not allow the meta-analyzes to have sufficient statistical weight and although they reported improvement in survival in a subgroup of patients ( advanced disease), even the residual disease and the clinical stage had a greater impact than the performance or not of lymphadenectomy.

In addition to this, we must not ignore that the realization of a lymphadenectomy carries a risk of greater morbidity, even in experienced centers, the main complications being: hemorrhage, formation of lymphoceles (although not everyone needs an invasive treatment for its resolution), vascular lesion, as well as a longer surgical time, need for transfusion and lymphedema development $(7-22 \%)(10,15)$. This made determining the real role of lymphadenectomy a difficult task.

With this in mind, it is interesting to mention that until 2 years ago the management of patients with advanced ovarian cancer, specifically whether or not to perform lymphadenectomy could only be based on non-ideal scientific studies (retrospectives, cohorts, etc.), due to the cost, feasibility and time needed to carry out a prospective randomized study, with subsequent biases and possibilities of error and the few randomized studies, had very important biases that prevented their results from being valid.

The LION study, a prospective, multicenter study, which included patients with stage III and IV ovarian cancer with negative clinical and intraoperative lymph node disease who underwent primary cytoreduction, leaving no residual disease (thus eliminating the main confounder of studies previous), excluding patients with suspicious lymph node disease (by palpation and inspection), the latter being the main bias of all previous studies. Patients were randomized intraoperatively to perform lymphadenectomy or not, with the outcome of overall survival. It should be noted that the quality of the surgeon was controlled and therefore of the surgery (an aspect that 
influences the outcome of these patients) (4).

Despite finding $57 \%$ of lymph node disease among patients who underwent lymphadenectomy, this did not impact overall survival (69.2 vs. 65.5 months) and progression-free survival of patients (25 months). However, the adverse events associated with lymphadenectomy were as described above and the increase in re-laparotomies and death was added within 60 days after surgery (4).

This study marks an important advance in the management of these patients, where the peritoneal disease will continue to determine the prognosis of this disease and where surgery with complete cytoreduction is a crucial part of the management of these patients (16), when reporting survivals greater than others. studies, showing the importance of a meticulous intra-abdominal surgery and where it will not be necessary to subject the patient to a procedure, in this case, lymphadenectomy, which could cause more harm than benefit by demonstrating that the latter does not have any therapeutic effect in this group of patients.

\section{Macroscopic lymph node involvement}

In patients with advanced ovarian cancer up to $41 \%$ will have macroscopic lymph node involvement during primary surgical exploration, so that lymph node resection will be part of primary surgery to achieve optimal cytoreduction, remembering that it is currently suggested that the goal is the complete resection of all visible disease (17). This radical vision of surgery in patients with advanced-stage has evolved in recent decades, where it has migrated from a surgery limited to the pelvis to one where the upper abdomen is approached and a residual disease smaller than $2 \mathrm{~cm}$ a leave without visible disease since a real impact on the survival of these patients was observed (18). It is known that the greatest tumor burden in patients with advanced ovarian cancer is intra-abdominal, on the peritoneal surfaces, causing the extent of surgery at these stages, is determined by the organs involved, in addition to the fact that it is this disease that determines the possibility of resection, residual disease, patient survival.

Determining if there is a macroscopic nodal disease is indispensable and difficult. Pre-operative imaging studies, such as computed tomography with contrast, which has a sensitivity between $48-80 \%$ and specificity of $67-83 \%$ for the detection of nodal disease, magnetic resonance imaging that has a sensitivity of $62.5 \%$ and Specificity of $83.5 \%$ and positron emission tomography (PET-CT) with a sensitivity of $75.5 \%$ to $83.5 \%$ and a specificity of $92.6 \%$ are useful for planning the surgical procedure, but all of them have limitations since it depends on the size of the lymph node and location of the disease $(19,20)$. Intraoperatively the evaluation of the disease varies according to the surgeon, since disease can be bulky that allows easy detection of the disease, however, if it is not, an adequate examination is necessary since it has been reported that palpation has low sensitivity and specificity and only $31 \%$ of lymph node disease can be detected and it has been suggested that it be discovered when opening the retroperitoneum, where it will increase the detection by $26 \%(2,5)$. Once identified, resection of the macroscopic lymph node disease should be performed as part of the cytoreductive surgery, since as mentioned previously and it was demonstrated in a retrospective study the resection of the macroscopic retroperitoneal disease will impact on survival of the patient by allowing patients to be left without the residual disease (12).

Finally, we are not only looking to resect the retroperitoneal macroscopic lymph node disease, but there are more and more reports about the importance and prognosis of mesenteric, celiac and cardiophrenic lymph node disease, which although they are not the objective of this paper, it speaks to us of the importance it has taken in these times to leave a patient with advanced ovarian cancer without visible disease, regardless of the location of the lymph node disease $(21,22)$.

\section{Histologic subtype}

The different histological subtypes of ovarian cancer are now recognized as clinical entities that differ from molecular level to clinical behavior, due to this the lymph node involvement varies between histological subgroups and tumor grade. High-grade serous carcinoma is the most common subtype $(70 \%)$, the lymph node condition varies according to the clinical stage; In the advanced stages, the condition can reach up to $67.5 \%$, so the arguments specified above apply perfectly to this histology (8). However, there are histologies such as mucinous carcinoma where it has been reported in multiple series that the lymph node condition is very low (0-7\%) so it has been suggested that lymphadenectomy can be omitted in these patients $(5,23)$.

\section{Conclusions}

Ovarian cancer is a complex disease, with multiple prognostic factors determined by the disease itself and by the treatment. In this review, we focused on a very specific 
aspect of the management of patients in the advanced stage and it was possible to confirm once again the importance of having quality scientific studies that allow us to make the best decisions in our patients. At this time, we can confidently say that systematic lymphadenectomy in patients with advanced ovarian cancer without clinically apparent nodal disease is not necessary, and the presence of macroscopic retroperitoneal lymph node disease will have to be resected as part of cytoreductive surgery since it will be this and the residual disease that determine the prognosis of the patients. Further advances on the knowledge of this disease will occur in the coming years thanks to the development of molecular markers and a greater knowledge of each histological subtype, which will allow us to more adequately determine the treatment of these patients, for the moment we know that mucinous subtype has a minimal lymph node affection, so lymphadenectomy can also be omitted in these patients.

\section{Acknowledgments}

Funding: None.

\section{Footnote}

Provenance and Peer Review: This article was commissioned by the Guest Editor (Heriberto Medina-Franco) for the series "Ovarian Cancer" published in Chinese Clinical Oncology. The article was sent for external peer review organized by the Guest Editor and the editorial office.

Conflicts of Interest: The author has completed the ICMJE uniform disclosure form (available at http://dx.doi. org/10.21037/cco-20-35). The series "Ovarian Cancer" was commissioned by the editorial office without any funding or sponsorship. The author has no other conflicts of interest to declare.

Ethical Statement: The author is accountable for all aspects of the work in ensuring that questions related to the accuracy or integrity of any part of the work are appropriately investigated and resolved.

Open Access Statement: This is an Open Access article distributed in accordance with the Creative Commons Attribution-NonCommercial-NoDerivs 4.0 International License (CC BY-NC-ND 4.0), which permits the noncommercial replication and distribution of the article with the strict proviso that no changes or edits are made and the original work is properly cited (including links to both the formal publication through the relevant DOI and the license). See: https://creativecommons.org/licenses/by-nc-nd/4.0/.

\section{References}

1. Prat J. Staging classification for cancer of the ovary, fallopian tube, and peritoneum. Int J Gynaecol Obstet 2014;124:1-5.

2. Harter P, Gnauert K, Hils R, et al. Pattern and clinical predictors of lymph node metastases in epithelial ovarian cancer. Int J Gynecol Cancer 2007;17:1238-44.

3. Morice P, Joulie F, Cammatte S, et al. Lymph node Involvement in epithelial ovarian cancer: Analysis of 276 pelvic and paraaortic lymphadenectomies and surgical implications. J Am Coll Surg 2003;197:198-205.

4. Harter P, Sehouli J, Lorusso D. et al. A randomized trial of lymphadenectomy in patients with advanced ovarian neoplasms. N Engl J Med 2019;380:822-32.

5. Eisenkop SM, Spirtos N. The clinical significance of occult macroscopically positive retroperitoneal nodes in patients with epithelial ovarian cancer. Gynecol Oncol 2001;82:143-9.

6. Podratz KC, Malkasian GD, Wleand HS, et al. Recurrent disease after negative second-look laparotomy in stages III and IV ovarian carcinoma. Gynecol Oncol 1988;29:274-82.

7. Burghardt E, Pickel H, Lahousen M, et al. Pelvic lymphadenectomy in operative treatment of ovarian cancer. Am J Obstet Gynecol 1986;155:315-9.

8. Di Re F, Baiocchi G, Fontanelli R, et al. Systematic pelvic and paraaortic lymphadenectomy for advanced ovarian cancer: Prognostic significance of node metastases. Gynecol Oncol 1996;62:360-5.

9. Fukasawa H, Kikkawa F, Tamakoshi K, et al. Lymphadenectomy instage III serous cystadenocarcinoma of the ovary. Int J Gynaecol Obstet 1995;51:239-45.

10. Panici PB, Maggioni An, Hacker N, et al. Systematic aortic and pelvic lymphadenectomy versus resection of bulky nodes only in optimally debulked advanced ovarian cancer: A randomized clinical trial. J Natl Cancer Inst 2005;97:560-6.

11. Chan JK, Urban R, Hu JM, et al. The potential therapeutic role of lymph node resection in epithelial ovarian cancer: a study of 13918 patients. Br J Cancer 2007;96:1817-22.

12. Du Bois A, Reuss A, Harter P, et al. Potential role of lymphadenectomy in advanced ovarian cancer: a combinen 
exploratory analysis of three prospectively randomized phase III multicenter trials. J Clin Oncol.2010;28:1733-9.

13. Kim HS, Ju W, Jee BC, et al. Systematic lymphadenectomy for survival in epithelial ovarian cancer. A Meta-Analysis. Int J Gynecol Cancer 2010;20:520-8.

14. Gao J, Yang X, Zhang Y. Systematic lymphadenectomy in the treatment of epithelial ovarian cancer: a metaanalysis of multiple epidemiology studies. Jpn J Clin Oncol 2015;45:49-60.

15. Trimbos JB. Staging of early ovarian cancer and the impact of lymph node sampling. Int J Gynecol Cancer 2000;10:8-11.

16. Eisenhauer E, Chi D. Ovarian cancer surgery- Heed this LIONs roar. N Engl J Med 2019;380:871-3.

17. Spirtos N, Gross G, Freddo J, et al. Cytoreductive surgery in advanced epithelial cancer of the ovary: the impact of aortic and pelvic lymphadenectomy. Gynecol Oncol 1995;56:345-52.

18. Du Bois A, Reuss A, Pujade-Lauraine E, et al. Role of surgical outcome as prognostic factor in advanced epithelial ovarian cancer: a combined exploratory analysis

Cite this article as: Salcedo-Hernández RA. Can lymphadenectomy be omitted in advanced ovarian cancer?-a brief review. Chin Clin Oncol 2020;9(4):46. doi: 10.21037/cco-20-35 of 3 prospectively randomized phase 3 multicenter trials. Cancer 2009; 115:1234-44.

19. Nam EJ, Yun MJ, Oh YT, et al. Diagnosis and staging of primary ovarian cancer: correlation between PETCT, Doppler US, and CT or MRI. Gynecol Oncol 2010;116:389-94.

20. Signorelli M, Guerra L, Pirovano C, et al. Detection of nodal metastases by $18 \mathrm{~F}-\mathrm{FDG}$ PET/CT in apparent early stage ovarian cancer: a prospective study. Gynecol Oncol 2013;131:395-9.

21. Baiocchi G, Cestari LA, Macedo MP, et al. Surgical implications of mesenteric lymph node metastasis from advanced ovarian cancer after bower resection. J Surg Oncol 2011;104:250-4.

22. Prader S, Wollmar N, Du Bois A, et al. Pattern and impact of metastatica cardiophrenic lymph nodes in advanced epithelial ovarian cancer. Gynecol Oncol 2019;152:76-81.

23. Salgado-Ceballos I, Rios J, Perez-Montiel D, et al. Is lymphadenectomy necessary in mucinous ovarian cancer? A single institution experience. Int J Surg 2017;41:1-5. 